\title{
Quantum Control of the Tin-Vacancy Spin Qubit in Diamond
}

\author{
Romain Debroux $\odot,{ }^{1}$ Cathryn P. Michaels $\odot,{ }^{1}$ Carola M. Purser, ${ }^{1,3}$ Noel Wan $\odot,{ }^{2}$ Matthew E. Trusheim,,${ }^{2,4}$ \\ Jesús Arjona Martínez®${ }^{1}$ Ryan A. Parker, ${ }^{1}$ Alexander M. Stramma, ${ }^{1}$ Kevin C. Chen, ${ }^{2}$ Lorenzo de Santis $\odot,{ }^{2,5}$ \\ Evgeny M. Alexeev, ${ }^{1,3}$ Andrea C. Ferrari $\odot,{ }^{3}$ Dirk Englund, ${ }^{2, *}$ Dorian A. Gangloff $\odot,{ }^{1, \dagger}$ and Mete Atatüre $\oplus^{1, \$}$ \\ ${ }^{1}$ Cavendish Laboratory, University of Cambridge, \\ JJ Thomson Avenue, Cambridge CB3 OHE, United Kingdom \\ ${ }^{2}$ Department of Electrical Engineering and Computer Science, Massachusetts Institute of Technology, \\ Cambridge, Massachusetts 02139, USA \\ ${ }^{3}$ Cambridge Graphene Centre, University of Cambridge, Cambridge CB3 OFA, United Kingdom \\ ${ }^{4}$ CCDC Army Research Laboratory, Adelphi, Maryland 20783, USA \\ ${ }^{5}$ QuTech, Delft University of Technology, P.O. Box 5046, 2600 GA Delft, Netherlands
}

(Received 24 May 2021; accepted 16 September 2021; published 30 November 2021)

\begin{abstract}
Group-IV color centers in diamond are a promising light-matter interface for quantum networking devices. The negatively charged tin-vacancy center $(\mathrm{SnV})$ is particularly interesting, as its large spin-orbit coupling offers strong protection against phonon dephasing and robust cyclicity of its optical transitions toward spin-photon-entanglement schemes. Here, we demonstrate multiaxis coherent control of the $\mathrm{SnV}$ spin qubit via an all-optical stimulated Raman drive between the ground and excited states. We use coherent population trapping and optically driven electronic spin resonance to confirm coherent access to the qubit at $1.7 \mathrm{~K}$ and obtain spin Rabi oscillations at a rate of $\Omega / 2 \pi=19.0(1) \mathrm{MHz}$. All-optical Ramsey interferometry reveals a spin dephasing time of $T_{2}^{*}=1.3(3) \mu \mathrm{s}$, and four-pulse dynamical decoupling already extends the spin-coherence time to $T_{2}=0.30(8) \mathrm{ms}$. Combined with transform-limited photons and integration into photonic nanostructures, our results make the $\mathrm{SnV}$ a competitive spin-photon building block for quantum networks.
\end{abstract}

DOI: 10.1103/PhysRevX.11.041041

Subject Areas: Quantum Information

\section{INTRODUCTION}

A light-matter quantum interface combines deterministic and coherent generation of single photons with a long-lived matter qubit [1-4]. This combination constitutes a foundational building block for quantum networking systems that exploit far-field radiation to generate remote entanglement and near-field interactions to realize nonlinear photonic gates [5-7]. Candidate systems include isolated atoms [8-11] and solid-state spins in the optical domain [12-14], as well as superconducting quantum circuits in the microwave regime [15]. An efficient quantum emitter is correspondingly well suited for light-assisted manipulation of its internal degrees of freedom [16-18]. The optical domain offers the critical advantage of wireless control fields which can be confined spatially to an optical wavelength, allowing

\footnotetext{
*englund@mit.edu

†ag50@cam.ac.uk

‡ma424@cam.ac.uk.
}

Published by the American Physical Society under the terms of the Creative Commons Attribution 4.0 International license. Further distribution of this work must maintain attribution to the author(s) and the published article's title, journal citation, and DOI. for selective control of individual systems on that length scale, and high-speed control arising from a high electric field density coupling to typically large electric dipole moments [19-22].

Diamond stands out as a particularly promising solidstate host for scalable fabrication of quantum light-matter interfaces [23], enabling all-optical control [24-27]. Within this material platform, the nitrogen-vacancy center (NV) has been used for pioneering quantum networking tasks owing to its excellent spin coherence [28-32]. Scaling up faces the challenge of improving its optical performance with tailored nanostructures [33], which remains difficult owing to the NV's sensitivity to nearby surfaces as a result of its permanent electric dipole moment [34,35]. In contrast, the group-IV color centers [36-47] are naturally compatible with photonic nanostructures owing to their inversion symmetry $[36,48]$ in which collection efficiencies exceeding 90\% [49] and large cavity-coupling efficiencies of more than $95 \%$ have been recently demonstrated [50-52]. Of these, the negatively charged silicon-vacancy center $(\mathrm{SiV})$ is the most studied, with demonstrations of coherent control of its ground state by microwave [53], alloptical [54], and acoustic [55] drive techniques. At millikelvin temperatures, where dephasing due to single-phonon scattering between orbital levels is suppressed, coherence 
times up to $13 \mathrm{~ms}$ [56] allow for more mature demonstrations of entanglement $[49,57,58]$. Building on these achievements, the recently reported tin-vacancy center $(\mathrm{SnV})[44-46,59]$ shares the desirable optical properties of SiV-large Debye-Waller factor of approximately 0.6 [60] with transform-limited photons [46]—and provides the additional advantages of (1) a long spin lifetime of $10 \mathrm{~ms}$ at $3.25 \mathrm{~K}$ (extrapolated to $>1 \mathrm{~s}$ at $1.7 \mathrm{~K}$ ) [46] and (2) optical cyclicity in the presence of an off-axis magnetic field or strain, which can allow for simultaneous highquality single-shot readout and efficient coupling to nuclear spins. These advantages stem from a large spin-orbit coupling, which suppresses decoherence due to singlephonon scattering in the ground state and establishes a common quantization axis between the ground and excited states, providing robust spin cyclicity. Conversely, the strong spin-orbit coupling also gives rise to orbitalforbidden spin transitions, which has limited microwavebased spin control [46] and may necessitate advanced microwave line engineering to achieve fast, coherent control of the $\mathrm{SnV}$ spin qubit.

In this paper, we demonstrate all-optical multiaxis coherent control of the $\mathrm{SnV}$ spin qubit by driving its efficient and coherent optical transitions with microwavemodulated laser fields. We demonstrate the flexibility of the all-optical approach by implementing coherent population trapping, optical Rabi driving, Ramsey interferometry, and dynamical decoupling of the $\mathrm{SnV}$ spin qubit. We further measure an electron-nuclear hyperfine-coupling strength of $42.6(4) \mathrm{MHz}$ for a spin-active $\mathrm{Sn}$ isotope. These results confirm the promise of $\mathrm{SnV}$ as a competitive nextgeneration light-matter quantum interface.

\section{COHERENT OPTICAL ACCESS TO THE SnV SPIN QUBIT}

The lilac frames in Fig. 1(a) illustrate the formation of the energy-level structure for the negatively charged $\mathrm{SnV}$ under the spin-orbit, Zeeman, and hyperfine couplings (see Supplemental Material [61] Sec. I). The strong spin-orbit coupling results in the ground- and excited-state manifolds having two orbital levels split by 850 and $3000 \mathrm{GHz}$, respectively [46], with a 484-THz (619-nm) optical transition energy between the manifolds (see Supplemental Material [61] Sec. I). An external magnetic field lifts the degenerate spin-orbit states via the Zeeman effect. Our qubit is defined as the Zeeman-split electronic spin states in the lower orbital branch $|1 \downarrow\rangle$ and $|2 \uparrow\rangle$. Spin-orbit interaction sets spin quantization along the $\mathrm{SnV}$ crystallographic symmetry axis for both ground and excited states. However, this axis pinning is weaker in the ground-state
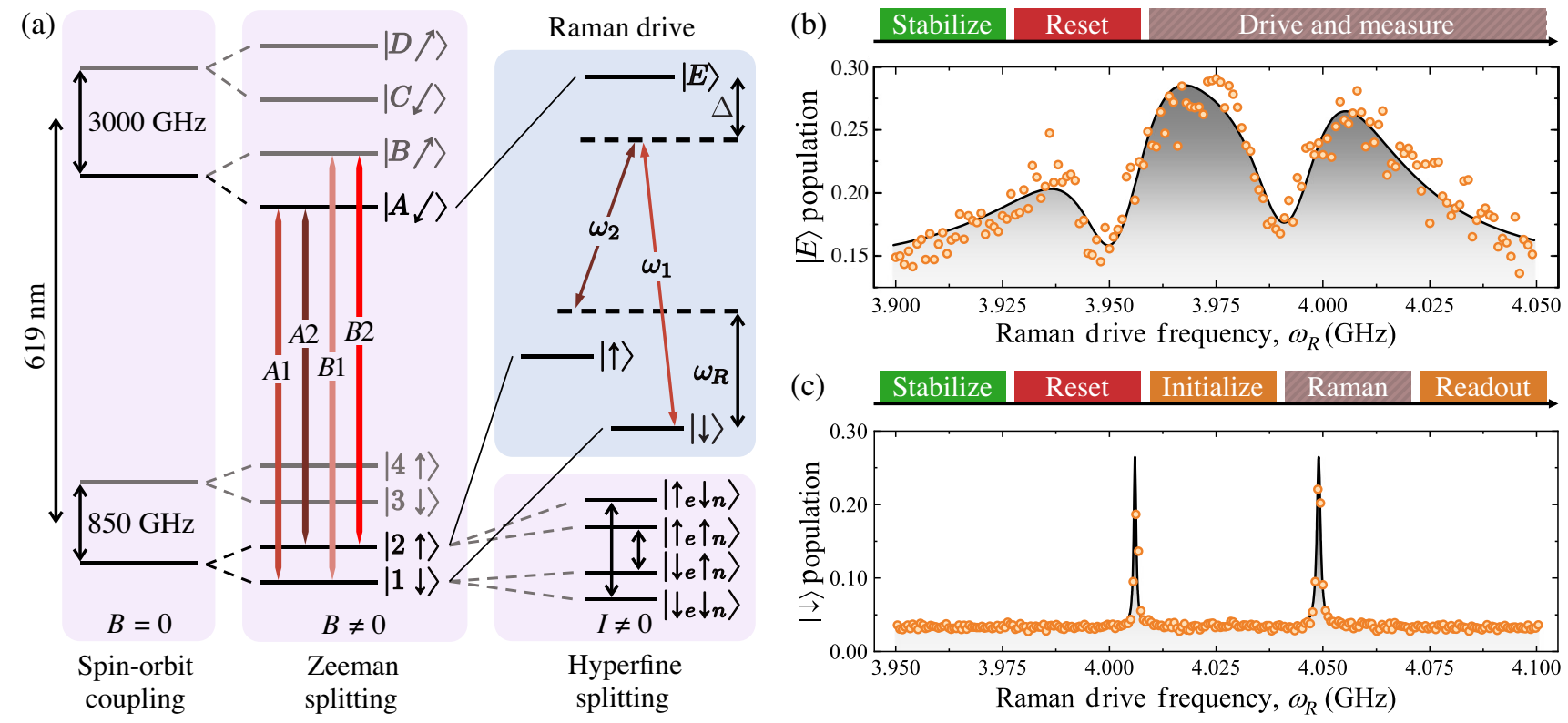

FIG. 1. SnV and Raman drive. (a) Lilac panels: energy levels of the SnV split by spin-orbit coupling (left), Zeeman splitting (middle), and hyperfine splitting (right). Blue panel: qubit defined by the $|\downarrow\rangle$ and $|\uparrow\rangle$ electronic levels. An optical lambda scheme is defined by two optical fields $\omega_{1}$ and $\omega_{2}$ detuned by $\Delta$ from the excited state $|E\rangle$. The Raman drive frequency $\omega_{R}=\omega_{1}-\omega_{2}$ is the energy offset between the two fields. (b) $|E\rangle$ population plotted in orange circles as a function of $\omega_{R}$ for $\Delta=0$ and 200 mT magnetic field strength applied at $54.7^{\circ}$ relative to the $\mathrm{SnV}$ symmetry axis. The pulse sequence consists of an approximately $10-\mu \mathrm{W}$ green stabilization pulse for $50 \mu \mathrm{s}, B 2$ reset pulse for $30 \mu \mathrm{s}$, and Raman drive with $p=40(4) \mathrm{nW}$ for $2 \mu \mathrm{s}$. The solid curve is a three-level model describing coherent population trapping. (c) $|\downarrow\rangle$ population plotted in orange circles as a function of $\omega_{R}$ for $\Delta=600 \mathrm{MHz}$ at 204-mT magnetic field strength. After the stabilize and reset pulses, the sequence includes an initialization pulse for $30 \mu \mathrm{s}$, a Raman drive with $p=40(4) \mathrm{nW}$ for $1 \mu \mathrm{s}$, and a readout pulse on $A 1$ for $30 \mu \mathrm{s}$. The solid curve is a two-Lorentzian fit resulting in an average linewidth of 900(200) $\mathrm{kHz}$ and spin resonances split by 42.6(4) $\mathrm{MHz}$. 
manifold, and its spin quantization axis can be perturbed by strain or magnetic field applied perpendicular to the $\mathrm{SnV}$ symmetry axis [37] with negligible impact on the excitedstate manifold. This mismatch between the quantization axes of the ground and excited spin states allows for the spin-cycling transitions ( $A 1$ and $B 2$ ) to achieve single-shot optical readout of the qubit, in tandem with the spinflipping transitions ( $A 2$ and $B 1$ ) to realize an optical lambda scheme (see Supplemental Material [61] Sec. I). The branching ratio between the spin-cycling and the spinflipping relaxation rates $\eta \approx 80$ measured for the $\mathrm{SnV}$ center studied in this work (see Supplemental Material [61] Sec. II) is consistent with a moderate-strain perturbation of 186(3) GHz (see Supplemental Material [61] Sec. I). The right-hand lilac frame displays the effect of hyperfine interaction. With $16.6 \%$ natural abundance among implanted ions, the Sn host atom can be a spin-active isotope $\left({ }^{115} \mathrm{Sn},{ }^{117} \mathrm{Sn}\right.$, or $\left.{ }^{119} \mathrm{Sn}\right)$, which couples the electronic spin qubit to the $I=1 / 2$ nuclear spin.

The light blue frame in Fig. 1(a) highlights how we leverage the $\mathrm{SnV}$ optical transitions to realize an optical lambda scheme between the $|1 \downarrow\rangle$ and $|2 \uparrow\rangle$ qubit states $(|\downarrow\rangle$ and $|\uparrow\rangle$, respectively) and the excited state $|E\rangle$. This is achieved by simultaneously driving the $A 1$ and $A 2$ transitions with lasers at frequencies $\omega_{1}$ and $\omega_{2}$, respectively, detuned relative to one another by the Raman frequency $\omega_{R}=\omega_{1}-\omega_{2}$. The Raman scheme is further detuned from the excited state by the single-photon detuning $\Delta$. For unpolarized light, the Rabi rate at which the spin is driven is then $\Omega=(1 / \sqrt{\eta})\left(p / p_{\text {sat }}\right)\left(\Gamma^{2} / 4 \Delta\right)$, where $p$ is the power in each of the optical fields driving $A 1$ and $A 2$, $p_{\text {sat }} \approx 5 \mathrm{nW}$ is our emitter's saturation power for the spincycling transition, and $\Gamma / 2 \pi=35 \mathrm{MHz}$ is the excited-state relaxation rate [62] (see Supplemental Material [61] Sec. III). Typical experimental values of order $p / p_{\text {sat }}=$ 10 and $\Delta / 2 \pi=300 \mathrm{MHz}$, and a measured $\eta=80(5)$ at 0.2-T magnetic field (see Supplemental Material [61] Sec. II) place the spin Rabi frequency in the $\mathrm{MHz}$ scale, which comfortably exceeds the inhomogeneous dephasing rate $1 / T_{2}^{*} \approx 1 \mu \mathrm{s}^{-1}$ [46], as required for coherent spin control. Driving the spin optically also causes a detuningdependent excited-state scattering rate $\Gamma_{\mathrm{OS}}=\left(p / p_{\text {sat }}\right) \times$ $\left(\Gamma^{3} / 8 \Delta^{2}\right)$ [62], which introduces a spin-relaxation rate $1 / T_{1, \mathrm{OS}}=\Gamma_{\mathrm{OS}} / \eta$ and a spin-dephasing rate $1 / T_{2, \mathrm{OS}}=\Gamma_{\mathrm{OS}}$ (see Supplemental Material [61] Sec. III). Maximizing the fidelity of a $\pi / 2$ gate with respect to $\Delta$, we find an optimal balance when $\Gamma_{\mathrm{OS}}=1 / T_{2}^{*}$ (see Supplemental Material [61] Sec. III).

Our $\mathrm{SnV}$ device consists of a nanopillar array fabricated into an $\mathrm{Sn}^{+}$ion-implanted diamond (see Supplemental Material [61] Sec. IV) and is cooled to $1.7 \mathrm{~K}$ in a magnetooptical cryostat (see Supplemental Material [61] Sec. V) [46]. Our all-optical measurement sequences include stabilization, reset, initialization, Raman drive, and readout pulses. The stabilize pulse uses a 532-nm laser to prevent blinking, which has been proposed to occur as a result of charge-state conversion of the $\mathrm{SnV}$ [60]. The initialize (reset) pulse consists of resonantly driving the $A 1$ (B2) transition, which polarizes the SnV spin into a $|\uparrow\rangle(|\downarrow\rangle)$ state in a time $\eta / \Gamma$ (approximately equal to $1 \mu \mathrm{s}$ ) via relaxation through the weakly allowed spin-flipping transition $A 2(B 1)$, achieving up to $99 \%$ initialization fidelities (see Supplemental Material [61] Sec. II). The reset pulse polarizes the population into the $|\downarrow\rangle$ state immediately before the initialization pulse, such that the fluorescence intensity of the initialization pulse corresponds to approximately $100 \%$ of the population. The fluorescence intensity of the readout pulse resonant on $A 1$ and normalized to that of the initialization pulse then provides a direct measurement of the population in the $|\downarrow\rangle$ state. The Raman drive encompasses all coherent pulse combinations we use in this work relying on a stimulated Raman process. For the optical pulses within the Raman drive, $\omega_{1,2}$ are realized using the two sidebands generated by passing a single-frequency laser through a microwave (MW)-modulated electro-optic modulator. The MW-modulation frequency splits the sidebands by $\omega_{R}$, the MW-modulation amplitude determines the power $p$ in each of the $\omega_{1,2}$ sidebands, and the MW-modulation phase dictates the relative phase between $\omega_{1,2}$ sidebands, the phase of the Raman drive $\phi$.

Figure 1(b) displays a coherent-population-trapping $(C P T)$ measurement as a first step to verify coherent optical access to the $\mathrm{SnV}$ spin qubit, where the $\omega_{1}$ and $\omega_{2}$ sidebands drive the spin-conserving and spin-flipping transitions, respectively, for $\Delta=0$. The top panel shows the $C P T$ pulse sequence, in which initialization, drive, and readout pulses are combined into one drive and measure step. The main panel presents the steady-state SnV fluorescence during the drive pulse as a function of $\omega_{R}$. We observe a broad feature, whose width is comparable to the excited-state linewidth $\Gamma$, accompanied by two narrower dips. A narrow dip in the $\mathrm{SnV}$ fluorescence spectrum (see Supplemental Material [61] Sec. VI) corresponds to the generation of a dark coherent superposition of the two ground states, $(1 / \sqrt{1+\eta})[|\downarrow\rangle-\sqrt{\eta}|\uparrow\rangle]$ (see Supplemental Material [61] Sec. VI) and is obtained when $\omega_{R}$ matches the spin-splitting frequency. In the presence of a spin-active $\mathrm{Sn}$ isotope $(I=1 / 2)$, the $C P T$ resonance splits into two dips arising from two nuclearspin-preserving transitions that are separated by the corresponding hyperfine-coupling rate. The $C P T$ spectrum in Fig. 1(b) indicates that the electronic spin qubit of the single $\mathrm{SnV}$ color center we measure, confirmed via intensity-correlation measurements (see Supplemental Material [61] Sec. VII) is indeed coupled to a spin-1/2 nuclear spin. Fitting a theoretical model (black curve) to the $C P T$ data, using a Lindbladian master-equation formalism (see Supplemental Material [61] Sec. VI) reveals a hyperfine-coupling strength of approximately $40 \mathrm{MHz}$ commensurate with previous reports on other group-IV 
color centers [40,53]. The depth of the two $C P T$ resonances confirms that the coherences of the spin ground states and the optical transitions are sufficient to implement coherent optical drive [40].

Having identified the two spin resonances via $C P T$, we move to the far-detuned stimulated Raman regime $\Delta \gg \Gamma$ to suppress scattering from the excited state during the coherent drive sequence. The top panel of Fig. 1(c) shows the pulse sequence, where the initialization, drive, and readout pulses are now separate operations. The main panel of Fig. 1(c) presents the population recovery of the $|\downarrow\rangle$ state as a function of $\omega_{R}$. When $\omega_{R}$ matches one of the electronic spin resonances, population from the initial $|\uparrow\rangle$ state is transferred to the $|\downarrow\rangle$ state, resulting in a peak in the $|\downarrow\rangle$ population. We fit our hyperfine-split double-peaked spectrum with two independent Lorentzian line shapes (solid curve), thereby allowing for high-precision measurement of the hyperfine constant for this $\mathrm{Sn}$ isotope, $A=$ 42.6(4) MHz. In the remainder of this work, we optically address a single-electronic-spin transition $\omega_{e}$ [defined as the lower energy peak in Fig. 1(c)], which sets the stage for its coherent control.

\section{MULTIAXIS COHERENT CONTROL}

We now demonstrate coherent spin control in the stimulated Raman regime. Figure 2(a) shows the Rabi oscillations of the population in the $|\downarrow\rangle$ state as we sweep the drive pulse duration $T$, with $\omega_{R}=\omega_{e}$. By fitting the data to a two-level model under a master-equation formalism (solid curve), we extract a Rabi rate of $\Omega / 2 \pi=$ 3.6(1) MHz (see Supplemental Material [61] Sec. III). Increased optical power straightforwardly yields Rabi rates of up to $\Omega / 2 \pi=19.0(1) \mathrm{MHz}$ (see Supplemental Material [61] Sec. VIII). This is an improvement of well over 3 orders of magnitude in spin Rabi frequency over direct microwave control realized thus far for SnVs [46] (see Supplemental Material [61] Sec. I). The inset shows the dependence of Rabi rate on power and detuning, with the expected linear dependence $\Omega \propto p / \Delta$. Our model further yields a total damping rate of 5.6(3) $\mu \mathrm{s}^{-1}$, as the sum of a depolarization ( $T_{1}$ process) rate of $0.28(2) \mu \mathrm{s}^{-1}$ and dephasing ( $T_{2}$ process) rate of 5.3(3) $\mu \mathrm{s}^{-1}$, with the latter in good agreement with the expected scattering rate $\Gamma_{\mathrm{OS}}=$ 3(1) $\mu \mathrm{s}^{-1}$ at a detuning of $\Delta / 2 \pi=1200 \mathrm{MHz}$. Finally, the model allows us to extract a $\pi / 2$-gate fidelity of $90.9(7) \%$ (see Supplemental Material [61] Sec. III C). The same scattering-induced dephasing mechanism sets an upper limit on the fidelity of all subsequent measurements involving more complex pulses. While the pulse fidelity achieved here remains modest, operating at larger detuning with increased laser power places high-fidelity gates within reach (see Supplemental Material [61] Sec. III C).

Full qubit control requires coherent drive about an arbitrary axis. Our approach realizes this via the combination
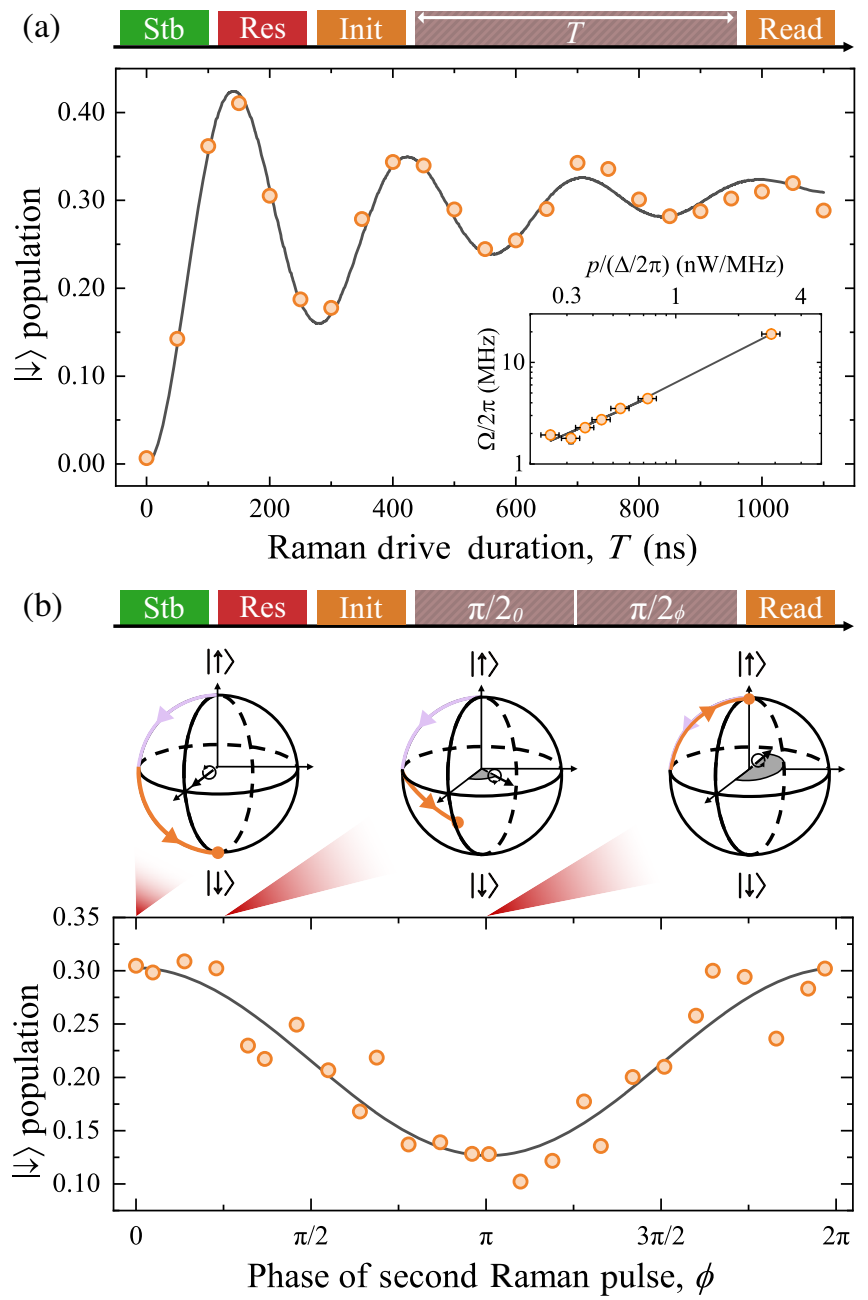

FIG. 2. Multiaxis coherent spin-qubit control. (a) $|\downarrow\rangle$ population (orange circles) as a function of the Raman drive duration $T$ with the pulse sequence shown at the top. The Raman drive is applied with $\Delta / 2 \pi=1.2 \mathrm{GHz}$ and $p=650(70) \mathrm{nW}$. The black curve is a fit to a two-level model under a master-equation formalism (see Supplemental Material [61] Sec. III). Inset: $\Omega / 2 \pi$ as a function of $p /(\Delta / 2 \pi)$ with a linear fit to the data (solid curve). Here, $\Delta / 2 \pi$ and $p$ are varied from 300 to $1200 \mathrm{MHz}$ and 40 to $650 \mathrm{nW}$, respectively. (b) Pulse sequence (top) with one $\pi / 2$ pulse about $x$ and a second about an axis rotated by an azimuthal angle $\phi$ from the $x$ axis. The $\pi / 2$ pulse duration is determined from Rabi measurements taken with $\Delta / 2 \pi=300 \mathrm{MHz}$ and $p=260(30) \mathrm{nW}$. Illustrated on the Bloch spheres are trajectories for $\phi=0$ (left), $\phi=\pi / 4$ (center), and $\phi=\pi$ (right). The $|\downarrow\rangle$ population (orange circles) is plotted as a function of $\phi$. The solid curve is a cosine function.

of the two-photon detuning, $\delta=\omega_{R}-\omega_{e}$, and $\phi$, the MW-controlled phase between the $\omega_{1,2}$ sidebands. The latter sets the control axis within the equatorial plane of the Bloch sphere, which is particularly relevant for implementing control sequences from the nuclear-magnetic-resonance toolbox $[63,64]$. We demonstrate this multiaxis control in Fig. 2(b) via a drive sequence comprising two $\pi / 2$ pulses, with the first driving the spin about the $x$ axis of the Bloch 
sphere $(\phi=0)$ and the second driving the spin about an axis rotated by an angle $\phi$ away from the $x$ axis. The population of the $|\downarrow\rangle$ state depends periodically on $\phi$ over the full $2 \pi$ range, where the cumulative drive for the maximum (minimum) $|\downarrow\rangle$ population corresponds to an effective $\pi(0)$ pulse. The phase dependence of the population readout confirms our ability to choose the quantum-state rotation axis.

\section{MEASURING SnV SPIN COHERENCE}

We use multiaxis coherent control to implement Ramsey interferometry in order to measure the inhomogeneous dephasing time $T_{2}^{*}$ of the $\mathrm{SnV}$ spin qubit. The top panel of Fig. 3(a) shows the corresponding pulse sequence comprised of two $\pi / 2$ pulses separated by a time delay $\tau$. We further impose a periodic recovery of the Ramsey signal by varying the rotation angle $\phi$ for the second $\pi / 2$ pulse as a function of $\tau$, such that $\phi=\tau \omega_{S}$ with the serrodyne frequency $\omega_{S} / 2 \pi=5 \mathrm{MHz}$. The main panel of Fig. 3(a) presents the dependence on $\tau$ and $\delta$ of the $|\downarrow\rangle$ population, which oscillates as a function of $\tau$ with a sum frequency given by $\omega_{\text {Ramsey }}=\omega_{S}+\delta+\Delta_{\mathrm{ac}}$, where $\Delta_{\mathrm{ac}}$ is the differential ac Stark shift. The latter term originates from the $|\downarrow\rangle$ state's stronger coupling to the Raman fields, and is only present during the Raman drive, thus acting as an effective detuning between the free precession rate of the spin and that of the drive's rotating frame (see Supplemental Material [61] Sec. IX). The period of the Ramsey fringes follows the expected $2 \pi / \omega_{\text {Ramsey }}$ behavior.

Figure 3(b) is an example line cut of the $|\downarrow\rangle$ population as a function of $\tau$ for a fixed $\delta / 2 \pi=-1 \mathrm{MHz}$. Fitting with the function $e^{-\left(\tau / T_{2}^{*}\right)^{2}} \sin \left(\omega_{\text {Ramsey }} \tau\right)$ yields $\omega_{\text {Ramsey }} / 2 \pi=$ 7.27(3) MHz, and hence, $\Delta_{\mathrm{ac}} / 2 \pi=3.3(5) \mathrm{MHz}$, comparable to the expected value (see Supplemental Material [61] Sec. VIII). We note that gate fidelity reduces the contrast in these measurements, but it does not affect the coherent spin precession between the two $\pi / 2$ pulses. The Gaussian envelope $e^{-\left(\tau / T_{2}^{*}\right)^{2}}$ provides an estimate of the spin inhomogeneous dephasing time $T_{2}^{*}$. By applying our model to the data for each $\delta$ in Fig. 3(a), we extract an average $T_{2}^{*}=1.3(3) \mu \mathrm{s}$. This is well within the range of expected inhomogeneous dephasing times limited by the naturally abundant ${ }^{13} \mathrm{C}$ nuclear spins in diamond $[46,56]$ and indicates that the $\mathrm{SnV}$ coherence is not phonon limited at $1.7 \mathrm{~K}$.

\section{IMPLEMENTING DYNAMICAL DECOUPLING}

To prolong the SnV spin-qubit coherence beyond the timescale set by the low-frequency magnetic noise of ${ }^{13} \mathrm{C}$ nuclei, we embed dynamical decoupling protocols within our optical pulse sequence, as illustrated in Fig. 4(a). We implement two example protocols: Hahn echo [65] comprising a single rephasing $\pi$ pulse about the $x$ axis (orange frame) and CPMG-2, a Carr-Purcell-Meiboom-Gill

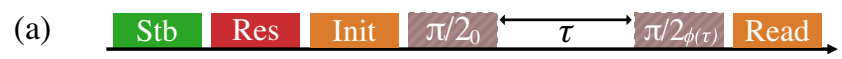

$|\downarrow\rangle$ population
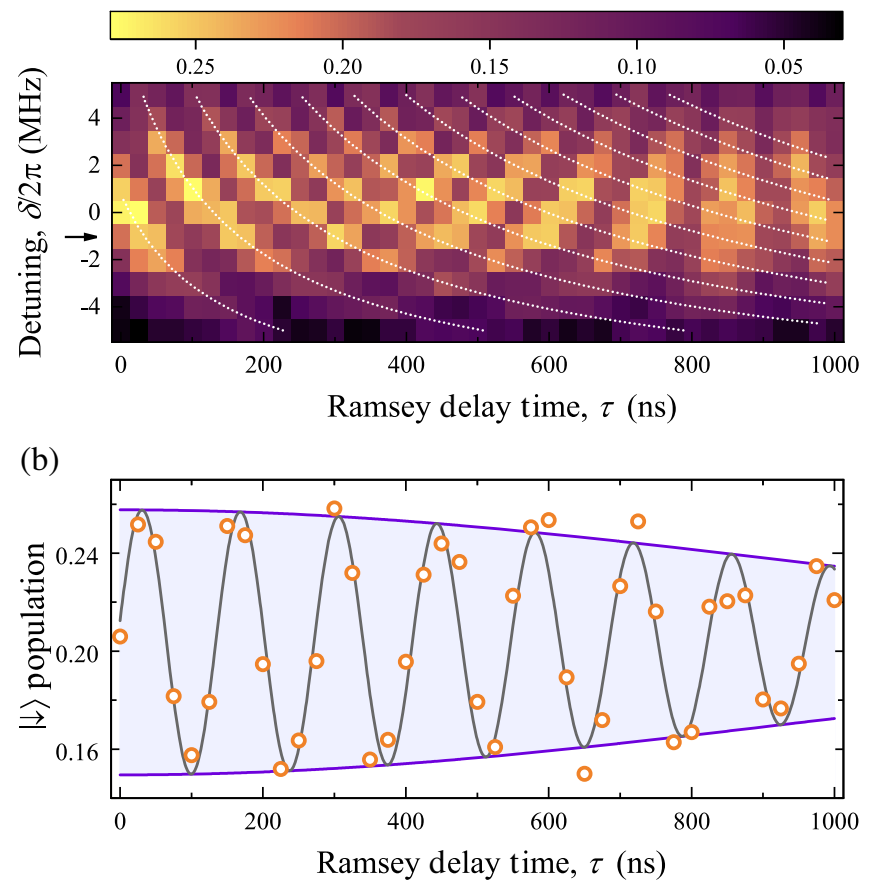

FIG. 3. Ramsey interferometry. (a) Ramsey pulse sequence with two $\pi / 2$ pulses separated by a delay time $\tau$ (top). The phase of the second pulse is swept according to $\phi=\tau \omega_{S}$, where $\omega_{S} / 2 \pi=5 \mathrm{MHz}$. The color indicates $|\downarrow\rangle$ population plotted as a function of $\tau$ and the two-photon detuning, $\delta$. Dotted white curves provide a guide to the eye for the expected $|\downarrow\rangle$ population recovery. Fits of the form $a \exp \left[-\left(\tau / T_{2}^{*}\right)^{2}\right] \sin \left(\omega_{\text {Ramsey }} \tau+\alpha\right)$ at each $\delta$ yield an average $T_{2}^{*}=1.3(3) \mu \mathrm{s}$ and $\Delta_{\mathrm{ac}} / 2 \pi=$ 3.3(5) $\mathrm{MHz}$ (see Supplemental Material [61] Sec. IX). (b) Line cut at $\delta / 2 \pi=-1 \mathrm{MHz}$ indicated by an arrow in (a), where the $|\downarrow\rangle$ population (orange circles) is measured as a function of $\tau$. The black curve corresponds to the fit described in (a), where $\omega_{\text {Ramsey }} / 2 \pi=7.27(3) \mathrm{MHz}, \quad T_{2}^{*}=1.4(3) \mu \mathrm{s}, \quad a=0.054(4)$, and $\alpha=0.16(9)$. The purple shaded region is bounded by the envelope $\pm a \exp \left[-\left(\tau / T_{2}^{*}\right)^{2}\right]$.

sequence [66] comprising two rephasing $\pi$ pulses about the $y$ axis (purple frame). Sweeping the phase of the final $\pi / 2$ pulse $\phi$ from 0 to $4 \pi$ and the decoupling delay time $\tau$ produces the two-dimensional maps of the $|\downarrow\rangle$ population in Fig. 4(a). The phase-dependent modulation of the Hahn echo signal lasts for approximately $30 \mu \mathrm{s}$, while the CPMG-2 signal extends significantly longer. Figure 4(b) presents the extracted visibility for the $\phi$-dependent modulations for both Hahn echo and CPMG-2 protocols as a function of the decoupling delay time $\tau$. Fitting the Hahn echo visibility (orange circles) as a function of $\tau$ with a stretched exponential function $\exp \left[-\left(\tau / T_{2}\right)^{n}\right]$ reveals an extended coherence time $T_{2}=28.3(6) \mu \mathrm{s}$. The exponent $n=3.6(3)$ is consistent with a noise spectrum from a slowly evolving nuclear-spin bath in diamond $[56,67]$. Applying the same fit function to the CPMG-2 visibility 


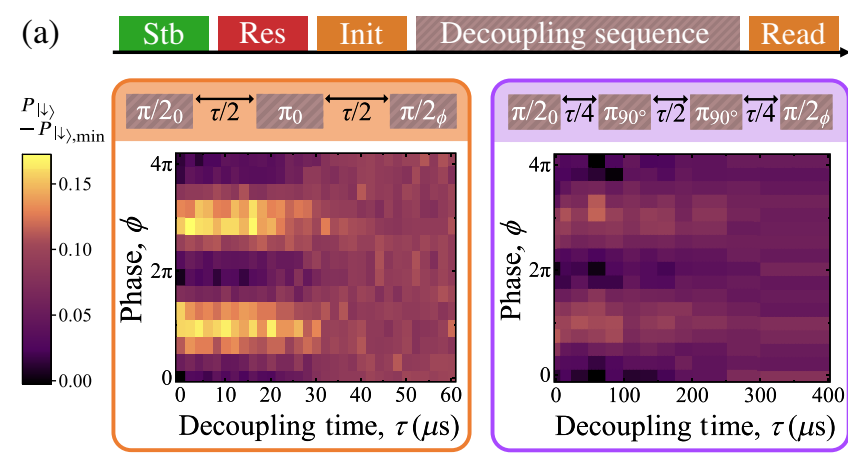

(b)

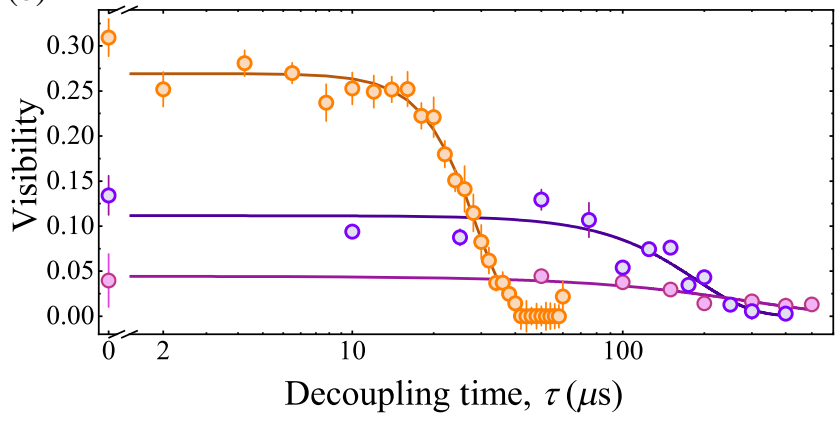

FIG. 4. Dynamical decoupling. (a) Decoupling pulse sequence (top) with two implementations: (left orange panel) Hahn echo, (right purple panel) CPMG-2. The phase $\phi$ of the second $\pi / 2$ pulse is variable. In both panels, color indicates the $|\downarrow\rangle$ population shown as a function of the total decoupling time $\tau$ and phase $\phi$. (b) Visibility $a / b$ obtained from fitting the function $a \cos (\phi)+b$ to the data shown in (a) at each delay time $\tau$ plotted as a function of $\tau$. Hahn echo data (orange circles) are fitted to the function $v_{0} \exp \left[-\left(\tau / T_{2}\right)^{n}\right]+v_{\infty}$, where $v_{0}=0.26(1)$, $v_{\infty}=0.013(5), n=3.7(4)$, and $T_{2}=28.3(6) \mu$ s (solid orange curve). CPMG-2 data (purple circles) are fitted to the same function with $v_{0}=0.11(1), n=2.1(9), T_{2}=0.19(2) \mathrm{ms}$, and $v_{\infty}$ is fixed to 0 (solid purple curve). CPMG-4 data (see Supplemental Material [61] Sec. X) are plotted (pink circles) and fit to the same function with $v_{0}=0.044(7), n=1.2(6)$, $T_{2}=0.30(8) \mathrm{ms}$, and $v_{\infty}$ is fixed to 0 (solid pink curve).

(purple circles), we find an improved coherence time of $T_{2}=0.19(2) \mathrm{ms}$ and $n=2.1(9)$. This coherence time is further extended by a CPMG-4 sequence (pink circles) to $T_{2}=0.30(8) \mathrm{ms}$, for which the envelope exponent is $n=1.2(6)$. This value for the exponent is compatible, within error bars, with an irreversible process $(n=1)$ or a quasistatic noise source $(n=2)$ from which further decoupling is possible, requiring further investigation. Despite this limitation, the tin qubit's CPMG-4 coherence time at $1.7 \mathrm{~K}$ is already within a factor of 5 of the best reported CPMG-4 coherence time for SiV at $100 \mathrm{mK}$ [56] and could be prolonged further with straightforward improvements of our gate fidelities.

\section{CONCLUSIONS AND OUTLOOK}

Our all-optical multiaxis coherent control of the SnV spin qubit establishes this alternative diamond color center as an attractive spin-photon quantum interface in the quest for efficient quantum networks. Carrying over the operational advantages that are common to the previously investigated group-IV color centers, such as a large Debye-Waller factor [36], transform-limited photon generation [46], and integration into photonic nanostructures enabled by their symmetry $[68,69]$, the $\mathrm{SnV}$ brings two additional advantages. First, the $\mathrm{SnV}$ spin remains competitive with the NV benchmark without requiring millikelvin operation temperature. Second, the strength of the spin-orbit interaction in the $\mathrm{SnV}$ offers the opportunity to simultaneously perform coherent spin control, single-shot readout, and nuclear-spin access all via the optical transitions. Our $\mathrm{SnV}$ spin-coherence time and Rabi rate can both be improved with stronger optical fields, which facilitates the suppression of optical scattering. Gate fidelities can be improved with technical refinements, and tailored pulse protocols are expected to result in 99.6\% gate fidelity for $\pi$ rotations [70]. An immediate next step toward realizing an efficient quantum memory is extending our all-optical approach to control the intrinsic $\mathrm{Sn}$ nuclear spin [71]. Further, integrating the $\mathrm{SnV}$ into photonic nanostructures $[44,50,51,68]$ will increase the photon-collection efficiency, and in parallel can strengthen the optical Rabi drive. Such structures should therefore enable efficient coherent control of an electronic spin coupled to a nuclear quantum memory with single-shot readout, a key building block for quantum networks $[5,6]$. Finally, going beyond an efficient local-area quantum network necessitates operation in the telecommunication bands. The similarity of the $\mathrm{SnV}$ wavelength to that of the $\mathrm{NV}$ makes all progress to date on efficient frequency conversion of $\mathrm{NV}$ photons to telecommunications bands $[72,73]$ readily applicable for the $\mathrm{SnV}$ as well.

\section{ACKNOWLEDGMENTS}

We acknowledge support from the ERC Advanced Grant PEDESTAL (Grant No. 884745), the EU Quantum Flagship 2D-SIPC, ERC grants Hetero2D and GSYNCOR, and EPSRC Grants No. EP/K01711X/1, No. EP/K017144/1, No. EP/N010345, and No. EP/L016087/1. R. D. acknowledges support from the Gates Cambridge Trust, C.P.M. from the EPSRC DTP, J. A. M. from the Winton Programme and EPSRC DTP, R.A.P. from the General Sir John Monash Foundation, and A.M.S. from EPSRC/NQIT. L. D. acknowledges funding from the European Union's Horizon 2020 research and innovation program under the Marie Sklodowska-Curie Grant Agreement No. 840393. M. T. acknowledges support through the Army Research Laboratory ENIAC Distinguished Postdoctoral Fellowship. K.C.C. acknowledges funding support by the National Science Foundation Graduate Research Fellowships Program and the NSF STC Center for Integrated Quantum Materials, NSF Grants No. DMR-1231319 and No. 1839155. N. W. acknowledges funding from the NSF 
Center for Ultracold Atoms and the NSF Center for Quantum Networks. D. A. G. acknowledges support from a St. John's College Title A Fellowship. D. E. acknowledges further support by the MITRE Quantum Moonshot Program.

R. D., C.P.M., and C.M.P. contributed equally to this work.

[1] W. B. Gao, A. Imamoglu, H. Bernien, and R. Hanson, Coherent Manipulation, Measurement and Entanglement of Individual Solid-State Spins Using Optical Fields, Nat. Photonics 9, 363 (2015).

[2] M. Atatüre, D. Englund, N. Vamivakas, S. Y. Lee, and J. Wrachtrup, Material Platforms for Spin-Based Photonic Quantum Technologies, Nat. Rev. Mater. 3, 38 (2018).

[3] D. D. Awschalom, R. Hanson, J. Wrachtrup, and B. B. Zhou, Quantum Technologies with Optically Interfaced Solid-State Spins, Nat. Photonics 12, 516 (2018).

[4] G. Wolfowicz et al., Quantum Guidelines for Solid-State Spin Defects, Nat. Rev. Mater. 6, 906 (2021).

[5] H. J. Kimble, The Quantum Internet, Nature (London) 453, 1023 (2008).

[6] S. Wehner, D. Elkouss, and R. Hanson, Quantum Internet: A Vision for the Road Ahead, Science 362, eaam9288 (2018).

[7] N. H. Lindner and T. Rudolph, Proposal for Pulsed On-Demand Sources of Photonic Cluster State Strings, Phys. Rev. Lett. 103, 113602 (2009).

[8] T. Wilk, S. C. Webster, A. Kuhn, and G. Rempe, SingleAtom Single-Photon Quantum Interface, Science 317, 488 (2007).

[9] C. W. Chou, H. de Riedmatten, D. Felinto, S. V. Polyakov, S. J. van Enk, and H. J. Kimble, Measurement-Induced Entanglement for Excitation Stored in Remote Atomic Ensembles, Nature (London) 438, 828 (2005).

[10] J. D. Thompson, T. G. Tiecke, N. P. de Leon, J. Feist, A. V. Akimov, M. Gullans, A. S. Zibrov, V. Vuletić, and M. D. Lukin, Coupling a Single Trapped Atom to a Nanoscale Optical Cavity, Science 340, 1202 (2013).

[11] J. Schupp, V. Krcmarsky, V. Krutyanskiy, M. Meraner, T. E. Northup, and B. P. Lanyon, Interface between Trapped-Ion Qubits and Traveling Photons with Close-to-Optimal Efficiency, PRX Quantum 2, 020331 (2021).

[12] P. Senellart, G. Solomon, and A. White, High-Performance Semiconductor Quantum-Dot Single-Photon Sources, Nat. Nanotechnol. 12, 1026 (2017).

[13] D. J. Christle et al., Isolated Spin Qubits in SiC with a HighFidelity Infrared Spin-to-Photon Interface, Phys. Rev. X 7, 021046 (2017).

[14] P. C. Humphreys, N. Kalb, J. P. J. Morits, R. N. Schouten, R. F. L. Vermeulen, D. J. Twitchen, M. Markham, and R. Hanson, Deterministic Delivery of Remote Entanglement on a Quantum Network, Nature (London) 558, 268 (2018).

[15] P. Magnard et al., Microwave Quantum Link between Superconducting Circuits Housed in Spatially Separated Cryogenic Systems, Phys. Rev. Lett. 125, 260502 (2020).

[16] E. Arimondo and G. Orriols, Nonabsorbing Atomic Coherences by Coherent Two-Photon Transitions in a
Three-Level Optical Pumping, Lett. Nuovo Cimento 17, 333 (1976).

[17] D. M. Meekhof, C. Monroe, B. E. King, W. M. Itano, and D. J. Wineland, Generation of Nonclassical Motional States of a Trapped Atom, Phys. Rev. Lett. 76, 1796 (1996).

[18] J. A. Gupta, R. Knobel, N. Samarth, and D. D. Awschalom, Ultrafast Manipulation of Electron Spin Coherence, Science 292, 2458 (2001).

[19] D. Press, T. D. Ladd, B. Zhang, and Y. Yamamoto, Complete Quantum Control of a Single Quantum Dot Spin Using Ultrafast Optical Pulses, Nature (London) 456, 218 (2008).

[20] J. H. Bodey, R. Stockill, E. V. Denning, D. A. Gangloff, G. Éthier-Majcher, D. M. Jackson, E. Clarke, M. Hugues, C. Le Gall, and M. Atatüre, Optical Spin Locking of a Solidstate Qubit, npj Quantum Inf. 5, 95 (2019).

[21] H. Levine, A. Keesling, A. Omran, H. Bernien, S. Schwartz, A. S. Zibrov, M. Endres, M. Greiner, V. Vuletić, and M. D. Lukin, High-Fidelity Control and Entanglement of Rydberg-Atom Qubits, Phys. Rev. Lett. 121, 123603 (2018).

[22] S. Debnath, N. M. Linke, C. Figgatt, K. A. Landsman, K. Wright, and C. Monroe, Demonstration of a Small Programmable Quantum Computer with Atomic Qubits, Nature (London) 536, 63 (2016).

[23] I. Aharonovich and E. Neu, Diamond Nanophotonics, Adv. Opt. Mater. 2, 911 (2014).

[24] D. A. Golter and H. Wang, Optically Driven Rabi Oscillations and Adiabatic Passage of Single Electron Spins in Diamond, Phys. Rev. Lett. 112, 116403 (2014).

[25] J. N. Becker, J. Grlitz, C. Arend, M. Markham, and C. Becher, Ultrafast All-Optical Coherent Control of Single Silicon Vacancy Colour Centres in Diamond, Nat. Commun. 7, 13512 (2016).

[26] B. B. Zhou, P. C. Jerger, V. O. Shkolnikov, F. J. Heremans, G. Burkard, and D. D. Awschalom, Holonomic Quantum Control by Coherent Optical Excitation in Diamond, Phys. Rev. Lett. 119, 140503 (2017).

[27] M. L. Goldman, T. L. Patti, D. Levonian, S. F. Yelin, and M. D. Lukin, Optical Control of a Single Nuclear Spin in the Solid State, Phys. Rev. Lett. 124, 153203 (2020).

[28] E. Togan et al., Quantum Entanglement between an Optical Photon and a Solid-State Spin Qubit, Nature (London) 466, 730 (2010).

[29] L. Childress and R. Hanson, Diamond NV Centers for Quantum Computing and Quantum Networks, MRS Bull. 38, 134 (2013).

[30] B. Hensen et al., Loophole-Free Bell Inequality Violation Using Electron Spins Separated by 1.3 Kilometres, Nature (London) 526, 682 (2015).

[31] C. E. Bradley, J. Randall, M. H. Abobeih, R. C. Berrevoets, M. J. Degen, M. A. Bakker, M. Markham, D. J. Twitchen, and T. H. Taminiau, A Ten-Qubit Solid-State Spin Register with Quantum Memory up to One Minute, Phys. Rev. X 9 , 031045 (2019).

[32] M. Pompili et al., Realization of a Multinode Quantum Network of Remote Solid-State Qubits, Science 372, 259 (2021).

[33] D. Riedel, I. Sollner, B. J. Shields, S. Starosielec, P. Appel, E. Neu, P. Maletinsky, and R. J. Warburton, Deterministic Enhancement of Coherent Photon Generation from a 
Nitrogen-Vacancy Center in Ultrapure Diamond, Phys. Rev. X 7, 031040 (2017).

[34] A. Faraon, C. Santori, Z. Huang, V. M. Acosta, and R. G. Beausoleil, Coupling of Nitrogen-Vacancy Centers to Photonic Crystal Cavities in Monocrystalline Diamond, Phys. Rev. Lett. 109, 033604 (2012).

[35] H. Bernien, L. Childress, L. Robledo, M. Markham, D. Twitchen, and R. Hanson, Two-Photon Quantum Interference from Separate Nitrogen Vacancy Centers in Diamond, Phys. Rev. Lett. 108, 043604 (2012).

[36] C. Bradac, W. Gao, J. Forneris, M. E. Trusheim, and I. Aharonovich, Quantum Nanophotonics with Group IV Defects in Diamond, Nat. Commun. 10, 5625 (2019).

[37] C. Heppetal., Electronic Structure of the Silicon Vacancy Color Center in Diamond, Phys. Rev. Lett. 112, 036405 (2014).

[38] T. Müller et al., Optical Signatures of Silicon-Vacancy Spins in Diamond, Nat. Commun. 5, 3328 (2014).

[39] B. Pingault, J. N. Becker, C. H. H. Schulte, C. Arend, C. Hepp, T. Godde, A. I. Tartakovskii, M. Markham, C. Becher, and M. Atatüre, All-Optical Formation of Coherent Dark States of Silicon-Vacancy Spins in Diamond, Phys. Rev. Lett. 113, 263601 (2014).

[40] L. J. Rogers et al., All-Optical Initialization, Readout, and Coherent Preparation of Single Silicon-Vacancy Spins in Diamond, Phys. Rev. Lett. 113, 263602 (2014).

[41] B.C. Rose et al., Observation of an Environmentally Insensitive Solid-State Spin Defect in Diamond, Science 361, 60 (2018).

[42] Z.-H. Zhang, P. Stevenson, G. Thiering, B. C. Rose, D. Huang, A. M. Edmonds, M. L. Markham, S. A. Lyon, A. Gali, and N.P. de Leon, Optically Detected Magnetic Resonance in Neutral Silicon Vacancy Centers in Diamond via Bound Exciton States, Phys. Rev. Lett. 125, 237402 (2020).

[43] P. Siyushev, M. H. Metsch, A. Ijaz, J. M. Binder, M. K. Bhaskar, D. D. Sukachev, A. Sipahigil, R. E. Evans, C. T. Nguyen, M. D. Lukin et al., Optical and Microwave Control of Germanium-Vacancy Center Spins in Diamond, Phys. Rev. B 96, 081201(R) (2017).

[44] A. E. Rugar, C. Dory, S. Aghaeimeibodi, H. Lu, S. Sun, S. D. Mishra, Z.-X. Shen, N. A. Melosh, and J. Vučković, Narrow-Linewidth Tin-Vacancy Centers in a Diamond Waveguide, ACS Photonics 7, 2356 (2020).

[45] A. E. Rugar, H. Lu, C. Dory, S. Sun, P. J. McQuade, Z.-X. Shen, N. A. Melosh, and J. Vučković, Generation of TinVacancy Centers in Diamond via Shallow Ion Implantation and Subsequent Diamond Overgrowth, Nano Lett. 20, 1614 (2020).

[46] M. E. Trusheim, B. Pingault, N. H. Wan, M. Gundogan, L. DeSantis, R. Debroux, D. Gangloff, C. Purser, K. C. Chen, M. Walsh et al., Transform-Limited Photons from a Coherent Tin-Vacancy Spin in Diamond, Phys. Rev. Lett. 124, 023602 (2020).

[47] M. E. Trusheim et al., Lead-Related Quantum Emitters in Diamond, Phys. Rev. B 99, 075430 (2019).

[48] G. Thiering and A. Gali, Ab Initio Magneto-Optical Spectrum of Group-IV Vacancy Color Centers in Diamond, Phys. Rev. X 8, 021063 (2018).

[49] M. K. Bhaskar et al., Experimental Demonstration of Memory-Enhanced Quantum Communication, Nature (London) 580, 60 (2020).
[50] K. Kuruma, B. Pingault, C. Chia, D. Renaud, P. Hoffmann, S. Iwamoto, C. Ronning, and M. Lončar, Coupling of a Single Tin-Vacancy Center to a Photonic Crystal Cavity in Diamond, Appl. Phys. Lett. 118, 230601 (2021).

[51] A. E. Rugar, S. Aghaeimeibodi, D. Riedel, C. Dory, H. Lu, P. J. McQuade, Z. X. Shen, N. A. Melosh, and J. Vuckovic, Quantum Photonic Interface for Tin-Vacancy Centers in Diamond, Phys. Rev. X 11, 031021 (2021).

[52] P. Fuchs, T. Jung, M. Kieschnick, J. Meijer, and C. Becher, A Cavity-Based Optical Antenna for Color Centers in Diamond, APL Photonics 6, 086102 (2021).

[53] B. Pingault, D.-D. Jarausch, C. Hepp, L. Klintberg, J. N. Becker, M. Markham, C. Becher, and M. Atatüre, Coherent Control of the Silicon-Vacancy Spin in Diamond, Nat. Commun. 8, 15579 (2017).

[54] J. N. Becker, B. Pingault, D. Groß, M. Gündoğan, N. Kukharchyk, M. Markham, A. Edmonds, M. Atatüre, P. Bushev, and C. Becher, All-Optical Control of the SiliconVacancy Spin in Diamond at Millikelvin Temperatures, Phys. Rev. Lett. 120, 053603 (2018).

[55] S. Maity et al., Coherent Acoustic Control of a Single Silicon Vacancy Spin in Diamond, Nat. Commun. 11, 193 (2020).

[56] D. D. Sukachev, A. Sipahigil, C. T. Nguyen, M. K. Bhaskar, R. E. Evans, F. Jelezko, and M. D. Lukin, Silicon-Vacancy Spin Qubit in Diamond: A Quantum Memory Exceeding 10 ms with Single-Shot State Readout, Phys. Rev. Lett. 119, 223602 (2017).

[57] R. E. Evans et al., Photon-Mediated Interactions between Quantum Emitters in a Diamond Nanocavity, Science 362, 662 (2018).

[58] C. T. Nguyen et al., Quantum Network Nodes Based on Diamond Qubits with an Efficient Nanophotonic Interface, Phys. Rev. Lett. 123, 183602 (2019).

[59] T. Iwasaki, Y. Miyamoto, T. Taniguchi, P. Siyushev, M. H. Metsch, F. Jelezko, and M. Hatano, Tin-Vacancy Quantum Emitters in Diamond, Phys. Rev. Lett. 119, 253601 (2017).

[60] J. Görlitz et al., Spectroscopic Investigations of Negatively Charged Tin-Vacancy Centres in Diamond, New J. Phys. 22, 013048 (2020).

[61] See Supplemental Material at http://link.aps.org/ supplemental/10.1103/PhysRevX.11.041041 for supporting measurements and further data analysis.

[62] C. J. Foot, Atomic Physics (Oxford University Press).

[63] A. Schweiger and G. Jeschke, Principles of Pulse Electron Paramagnetic Resonance (Oxford University Press, Oxford, 2001).

[64] A. Abragam, The Principles of Nuclear Magnetism (Oxford University Press, Oxford, 1983).

[65] E. L. Hahn, Spin Echoes, Phys. Rev. 80, 580 (1950).

[66] H. Y. Carr and E. M. Purcell, Effects of Diffusion on Free Precession in Nuclear Magnetic Resonance Experiments, Phys. Rev. 94, 630 (1954).

[67] L. Childress, M. V. G. Dutt, J. M. Taylor, A. S. Zibrov, F. Jelezko, J. Wrachtrup, P. R. Hemmer, and M. D. Lukin, Coherent Dynamics of Coupled Electron and Nuclear Spin Qubits in Diamond, Science 314, 281 (2006).

[68] N. H. Wan et al., Large-Scale Integration of Artificial Atoms in Hybrid Photonic Circuits, Nature (London) 583, 226 (2020). 
[69] C. T. Nguyen et al., An Integrated Nanophotonic Quantum Register Based on Silicon-Vacancy Spins in Diamond, Phys. Rev. B 100, 165428 (2019).

[70] E. Takou and S. E. Economou, Optical Control Proocols for High-Fidelity Spin Rotations of Single $\mathrm{SiV}^{-}$and $\mathrm{SnV}^{-}$ Centers in Diamond, Phys. Rev. B 104, 115302 (2021).

[71] C. P. Michaels et al., Multidimensional Cluster States Using a Single Spin-Photon Interface Coupled Strongly to an Intrinsic Nuclear Register, Quantum 5, 565 (2021).
[72] R. Ikuta et al., Frequency Down-Conversion of $637 \mathrm{~nm}$ Light to the Telecommunication Band for Non-Classical Light Emitted from NV Centers in Diamond, Opt. Express 22, 11205 (2014).

[73] A. Dréau, A. Tcheborateva, A. E. Mahdaoui, C. Bonato, and R. Hanson, Quantum Frequency Conversion of Single Photons from a Nitrogen-Vacancy Center in Diamond to Telecommunication Wavelengths, Phys. Rev. Applied 9, 064031 (2018). 